\title{
A prospective study of HLA*B-5801 genotyping in preventing allopurinol- induced severe cutaneous adverse reactions
}

\author{
Tai-Ming Ko ${ }^{1 *}$, Jer-Yuarn $\mathrm{Wu}^{2}$, Yuan-Tsong Chen ${ }^{2}$, Chen-Yang Shen ${ }^{2}$ \\ From 6th Drug Hypersensitivity Meeting (DHM 6) \\ Bern, Switzerland. 9-12 April 2014
}

\section{Background}

Allopurinol, a commonly prescribed medication for gout and hyperuricemia, is a frequent cause of severe cutaneous adverse reactions (SCAR), which include the hypersensitivity syndrome, Stevens-Johnson syndrome, and toxic epidermal necrolysis. Allopurinol-induced SCAR is strongly associated with the HLA-B*58:01 allele. We sought to prevent allopurinol-induced SCAR by using HLA-B*58:01 screening to prospectively identify subjects at risk for this condition.

\section{Methods}

From 14 hospitals in Taiwan, we recruited 2037 candidate subjects who had indication for allopurinol treatment but had not taken allopurinol previously. We genotyped DNA purified from the subjects' $\mathrm{PBMC}$ to determine whether they carried the HLA-B*58:01 allele. Those testing positive for HLA-B*58:01 (20.57\% of the total) were advised not to take allopurinol and were given an alternative medication or advised to continue taking their pre-study medication; those testing negative $(79.43 \%)$ were given allopurinol. We interviewed the subjects by telephone once a week for 3 months to monitor them for symptoms. We used the estimated historical incidence of SCAR for comparison.

\section{Results}

Mild, transient rash developed in $4.63 \%$ of subjects during follow-up; more widespread rash was observed in $0.13 \%$ of subjects, who were hospitalized. SCAR did not develop in any of the HLA-B*58:01-negative subjects receiving allopurinol; this is in contrast to the 4 expected cases of SCAR based on the estimated historical incidence of

${ }^{1}$ Institute of Biomedical Sciences, Academia Sinica, Taipei, Taiwan, Taiwan Full list of author information is available at the end of the article allopurinol-induced SCAR (0.29\%) ( $\mathrm{p}$ value=0.0266; Fisher's two-tailed exact tests).

\section{Conclusions}

The identification of subjects carrying the HLA-B*58:01 allele and the avoidance of allopurinol therapy in these subjects were strongly associated with a decreased incidence of allopurinol-induced SCAR.

\section{Funding}

Funded by the Academia Sinica Genomic Medicine Multicenter Study and National Science Council of Taiwan.

\section{Authors' details}

${ }^{1}$ Institute of Biomedical Sciences, Academia Sinica, Taipei, Taiwan, Taiwan.

${ }^{2}$ Academia Sinica, Institute of Biomedical Sciences, Taiwan.

Published: 18 July 2014

doi:10.1186/2045-7022-4-S3-O4

Cite this article as: Ko et al:: A prospective study of HLA*B-5801 genotyping in preventing allopurinol-induced severe cutaneous adverse reactions. Clinical and Translational Allergy 2014 4(Suppl 3):04.

Submit your next manuscript to BioMed Central and take full advantage of:

- Convenient online submission

- Thorough peer review

- No space constraints or color figure charges

- Immediate publication on acceptance

- Inclusion in PubMed, CAS, Scopus and Google Scholar

- Research which is freely available for redistribution 